\title{
La seguridad humana: del concepto al enfoque. Causas de la reducción de su uso como concepto NuRia Hernández Garcí́ ${ }^{*}$
}

\section{RESUMEN}

El concepto de seguridad humana, si bien lo que pretendía era desligarse del concepto de seguridad de guerra fría, ligado al armamento y poner su centro en la persona, no ha tenido demasiado éxito en la esfera internacional. Este artículo realizará una evolución del concepto de seguridad humana y se verá cuál es su vinculación con la protección de derechos humanos y otros principios de Naciones Unidas como la responsabilidad de proteger. Este artículo propondrá que la falta de concreción del término, su uso selectivo y la unión de perspectivas de derechos humanos y seguridad, es lo que le ha restado poder.Y, así, como ha pasado con otros términos de construcción política, su politización ha llevado a:a) que se necesite un cambio en el uso del concepto como tal, convirtiéndose en un modo de hacer, esto es, un enfoque amplio que proponga marcos de análisis y planificación; $y, b$ ) que hayan aparecido otros conceptos que se usen más en detrimento del de la seguridad humana como el de resiliencia, que pone el foco en la población que hay que proteger a través del empoderamiento.

\section{Palabras clave}

Seguridad humana; derechos humanos; responsabilidad de proteger; seguridad militar; desarrollo sostenible.

\section{TITLE}

Human Security: from the concept to the approach. Why it's not used as a concept anymore?

\section{Abstract}

The concept of human security was thought as an alternative for the traditional and military security concept that was used both before and during the ColdWar. Its objective was to focus on the wellbeing of people, however, it has been proved not being successful enough. This paper aims to explore the evolution of the concept of human security and its relationship with human rights' protection and other principles used by the United Nations, as the responsibility to protect. This paper will propose that the cause for not using the concept has been both the lack of a clear definition of the concept and its selective use by politicians; and, also, mixing human rights with traditional security approaches. Therefore, and exactly as the same as has happened to other political constructions, its politicization has led to: a) the need for a change in the use of the concept that has turn into an approach; and, b) other concepts have arisen such as resilience, which implies an active participation of the population.
\end{abstract}

\section{KEYWORDS}

Human security; human rights; responsibility to protect; military security; sustainable development..

\section{DOI:}

https://doi.org// 0.15366/relacionesinternacionales2020.43.002

Formato de citación recomendado:

HERNÁNDEZ GARCÍA, Nuria. "La seguridad humana: del concepto al enfoque. Causas de la reducción de su uso como concepto”, en Relaciones Internacionales, n 43, 2020, pp. 33-48.

* Nuria HERNÁNDEZ GARCÍA, Doctoranda FPU de segundo año en el programa de Estudios Europeos y Relaciones Internacionales en la UCH-CEU. Licenciada en Ciencias Políticas y Periodismo y con un Master en Relaciones Internacionales por la UPC ha realizado dos estancias de investigación en COMPAS (University of Oxford). Sus áreas de interés son: integración, refugio y asilo, Unión Europea, derechos humanos, seguridad.

Recibido: 29/09/2019

Aceptado: 19/12/2019 


\section{$\mathrm{I}$}

\section{ntroducción}

"Un uso descuidado y un abuso del concepto se ha demostrado inútil para todos menos para los políticos"'. Así hablaba David Baldwin del concepto de seguridad. Es necesario para una comprensión del término "seguridad humana", objetivo de este artículo, entender qué es seguridad, cómo ha evolucionado hasta desarrollar el concepto de seguridad humana, los vínculos de este último con los derechos humanos y el de responsabilidad de proteger y se intentará dar respuesta a por qué se ha dejado de usar como concepto en sí.

Los objetivos del presente artículo son, por tanto: primero, explorar el concepto de seguridad; segundo, analizar el contenido del concepto de seguridad humana; tercero, señalar la relación entre seguridad humana, derechos humanos y responsabilidad de proteger; $y$, cuarto, identificar las posibles causas para que el concepto de seguridad humana haya perdido fuerza como tal.

Para ello, se seguirá una estructura de dos bloques principales usando una metodología expositiva y descriptiva teleológica. En la primera parte se hablará de los objetivos uno y dos y en la segunda parte del objetivo tres. Así pues, primero se realizará una exploración a través exclusivamente de la revisión bibliográfica desde una perspectiva histórica de la definición de seguridad. Después, se procederá a un análisis de cómo la falta de definición de la palabra seguridad afecta a la delimitación de lo que es la seguridad humana a partir de documentación académica y del Programa de Naciones Unidas para el Desarrollo (PNUD) ${ }^{2}$. Para continuar con la aproximación al objeto que nos ocupa, que es la seguridad humana, se señalará y se profundizara en su contenido en cuanto a su relación con los derechos humanos y otros principios de Naciones Unidas que buscan la protección de las personas como el de la responsabilidad de proteger.

Por último, en el apartado de las conclusiones, se identificarán las posibles causas del cambio en el uso del concepto de seguridad humana. Así pues, se concluirá que ya no se utiliza como concepto en sí, sino como enfoque para responder a las amenazas, de lo más diversas, que puedan afectar a la vida de las personas de manera que puedan vivir libres de temor, con dignidad y sin miseria. Fruto de este análisis se tratará de valorar el impacto que supone el cambio de concepto a enfoque y se sugerirán algunas ideas para enfrentarse a la indefinición del concepto.

\section{Origen del concepto "seguridad humana"}

¿Qué se entiende por seguridad? Un concepto que se utiliza para justificar la suspensión de libertades civiles, declarar la guerra y disponer masivamente de los recursos de una manera u otra durante todo el siglo $\mathrm{XX}$, y siglo $\mathrm{XXI}^{3}$ debería estar definido. Sin embargo, esto no es así. Es un concepto que ha estado abandonado y que, aún después de la Guerra Fría cuando trataban

\footnotetext{
BALDWIN, David A.,"The concept of security” en Review of International Studies, n² 23, I997, pp. 5-26.

2 Existen numerosos documentos de Naciones Unidas que tratan los conceptos de seguridad humana y también el de responsabilidad de proteger. Sin embargo, y a efectos de hacer el texto más comprensible al lector, solo van a utilizarse los imprescindibles para seguir el hilo conductual del argumento. Por ello se han seleccionado los textos del PNUD y los informes más relevantes que dieron origen a ambos conceptos y su evolución. Si se quieren consultar otros documentos sobre seguridad humana tanto académicos como de Naciones Unidas ir a: https://www.un.org/humansecurity/publications-on-human-security/ [consultado el I de febrero de 2020].

3 Cabe resaltar que aunque el concepto apareció teóricamente en los noventa a consecuencia del contexto que se menciona, ha seguido desarrollándose en el siglo $\mathrm{XXI}$ donde la situación sigue siendo la misma y se sigue sin contar con una definición precisa del término seguridad.
} 
de "redefinir"4 el concepto de seguridad, pocos han sido los académicos que han tratado de explicarlo. De hecho, Baldwin afirma que no es descabellado asegurar que el análisis conceptual de seguridad empezó y terminó con el artículo de Arnold Wolfers en 1952 titulado National Security as an Ambiguous Symbol 5 . La importancia de las palabras, y de definirlas, especialmente en la Ciencia Política, reside en que ésta es la "manera efectiva de resolver problemas sustantivos de investigación"'. Sin embargo, clarificar el concepto, en este caso el de seguridad, no sería suficiente para formular teorías o desarrollar marcos teóricos, es decir, no los sustituye, aunque es un paso previo para desarrollarlos ${ }^{7}$.

El arduo ejercicio de definir "seguridad" sirve primero para saber qué es; después promueve un análisis racional de las políticas que llevan su nombre, con la comparación de un tipo de política de seguridad con otra; y, más adelante, facilita un terreno común donde los expertos y académicos pueden comunicarse. Barry Buzan y Richard Ullman mezclan el análisis conceptual con el empírico, hablando de las condiciones necesarias para que haya seguridad y de qué sería no tenerla respectivamente, pero Baldwin señala, con acierto, que la clarificación de un concepto precede a la búsqueda de las determinadas condiciones para que se dé ese concepto, ya que la identificación de esas condiciones presupone que se conoce el término que se estudia ${ }^{8}$. Lo cierto es que, dejar en la definición espacio para la investigación empírica es importante, porque efectivamente, dependerá de las circunstancias respondiendo a preguntas como: seguridad para quién, de qué amenazas, protegiendo qué, cuánta seguridad es necesaria ${ }^{9} .$. Es sin duda un concepto amplio, multidimensional y vivo.

Con lo anterior, se pretende mostrar la complejidad de la situación. El término seguridad no está apenas definido, por lo menos no con una definición consensuada y aceptada internacionalmente a nivel académico y político, por lo tanto, el término seguridad humana presenta una batalla de segundo nivel en cuanto a su delimitación. Esto ya deja entrever los problemas que se plantean en este artículo derivados del uso de un concepto que, en su esencia, no está definido con claridad y consenso. Para acercarnos al concepto de seguridad humana, se procederá a un breve repaso del concepto de seguridad en su sentido tradicional, pero sin entrar excesivamente en los autores que lo han estudiado, ni en la evolución del mismo en la teoría política a lo largo de la historia.

\section{I Seguridad en el sentido tradicional}

Sin profundizar en la evolución histórica del concepto $^{10}$, resulta interesante pararse a analizar cómo evoluciona de ser una característica del individuo a un bien público, y, cómo a partir de ahí se identifica con la seguridad con respecto a otros estados, adquiriendo ese carácter militar que

\footnotetext{
4 Las comillas son uso de la autora, puesto que como se verá en la literatura hay voces que critican precisamente la falta de definición del concepto de seguridad, por lo que volver a definir algo, redefinir algo, que no ha sido definido desde un principio resulta una tarea de éxito dudoso.

5 Ibídem, p. 23.

6 OPPENHEIM, Felix E., "The language of political inquiry: problems of clarification" en GREENSTEIN, Fred y POLSBY, Nelson (eds.), Political Science: Scope and Theory,Addison-Wesley, Reading, 1975, p. 284.

BALDWIN, David A., "The concept...", op.cit., p. 7.

8 Ibídem, pp. 7-8.

9 lbíd., pp. 24-25.

10 Para una mayor comprensión del concepto y de su evolución histórica ver: ROTHSCHILD, Emma, "What is security?” en Daedalus, vol. I 24, $\mathrm{n}^{\circ}$ 3, 1995, pp. 53-98; PÉREZ DE ARMIÑO, Karlos y MENDIA AZKUE, Irantzu (eds.), Seguridad Humana, aportes críticos al debate teórico y político, Tecnos, Madrid, 2013.
} 
tendrá su máximo exponente en la Guerra Fría.

Las corrientes filosóficas y políticas sobre qué es seguridad han estado presentes en el debate europeo desde las crisis del siglo XVII. También es un concepto que ha evolucionado inclinándose, según la época y autores, hacia ser considerado un bien más individual o del estado. En el pensamiento liberal tradicional, la seguridad se entiende como un bien individual, que responde al derecho de los individuos a no tener miedo de sufrir daños personales. Durante la Revolución Francesa, la seguridad se sigue entendiendo como un derecho privado, de cada individuo, pero que tiene que estar garantizado por la sociedad, a través del pacto o contrato social. Así, Locke, Rousseau, Montesquieu, Kant y Condorcet"', difiriendo en sus más y sus menos, sometían la seguridad individual a la seguridad de la nación. Esto se hace cuando se identifica el pacto social como resultado de la búsqueda de esa seguridad del individuo, y, por tanto, es el estado, quien tiene que garantizar esa seguridad de sus individuos y se habla de la seguridad de la nación, o seguridad del estado frente a otros estados. De hecho, uno de los elementos fundacionales del carácter social del ser humano es la preocupación por la seguridad, como señalaban Hobbes o Locke, $y$, efectivamente, desde el mismo momento del nacimiento del estado la seguridad ha sido uno de los elementos centrales en los ámbitos de la política y de la sociedad ${ }^{12}$.

La sociedad y el estado han cambiado a lo largo de la historia del hombre. Pero la seguridad, la búsqueda de la protección y la defensa de lo propio han estado presentes en la naturaleza innata del hombre que pertenece a una comunidad. A partir del momento en el que el estado se entiende constituido por población, territorio, gobierno y soberanía según Westfalia ${ }^{13}$, y es el único actor protagonista en las Relaciones Internacionales, defenderse de otros estados es la principal preocupación de la agenda de seguridad del estado: controlar, a través del monopolio del uso de la fuerza ${ }^{14}$, la seguridad interna y externa. Efectivamente, la seguridad nacional tiene dos dimensiones, una interna y otra de carácter externo, según cómo se enfoque la política de seguridad. Precisamente la definición del concepto sirve para saber cómo enfocar esa política y promover un análisis racional de las políticas que llevan su nombre con la comparación de un tipo de política de seguridad con otra y facilitar un terreno común donde los expertos y académicos pueden comunicarse ${ }^{15}$.

Para proceder a la definición del concepto, muchos autores hablan de la Guerra Fría como punto de inflexión, como se verá a continuación. Antes de la Guerra Fría, la seguridad se entendía en términos militares, donde el estado era el actor principal y se identificaba como amenaza cualquiera que pusiera en peligro su identidad, autonomía o territorio. Es un concepto de carácter nacional, centrado en la defensa de lo territorial y a través de los medios militares, desde una perspectiva exclusivista, entendiéndose que cada estado "trata de garantizar su propia seguridad como medio de asegurar con ello la de sus propios ciudadanos prescindiendo en buena medida

\footnotetext{
I" ROTHSCHILD, Emma, “What is...”, op.cit., pp. 62-65.

I2 AGUIAR MOLINA, Pablo y ALCALDE VILLACAMPA, Javier, “Seguridad Humana. Un marco de análisis" en Conflictos, política y derecho, 20I I, PP. $21-52$.

13 Se refiere al Tratado de Westfalia de 1648, hito que marca las bases de la nación/estado soberana.

14 Este monopolio del uso de la fuerza lo introduce en la formula westfaliana del estado, el sociólogo Max Weber en su ensayo Politics as a Vocation de 1919.

15 BALDWIN, David A., “The concept...”, op.cit., p. 26.
} 
de los efectos que su propia conducta pueda tener en la seguridad de los otros estados"16.

Durante la Guerra Fría el mundo estaba dividido en dos bloques, el capitalista liderado por Estados Unidos (EEUU) y el comunista, liderado por la Unión de Repúblicas Soviéticas (URSS). La preocupación en las Relaciones Internacionales era el equilibro de poder entre ambos y la amenaza nuclear y de control e influencia sobre determinadas zonas del globo. La seguridad se centraba, en el primer mundo, en el estado, entendiéndose como seguridad nacional, y en palabras de Hans Morgenthau "integridad del territorio nacional y sus instituciones"17 y, en buena medida, militar. En el mundo en vías de desarrollo también es el estado el que se sitúa en el centro de la seguridad, así como los regímenes políticos.

La clásica definición de ArnoldWolfers antes del fin de la Guerra Fría es el punto de partida para definir la seguridad como la ausencia de amenazas a unos valores subjetivos que diferirán según la política de seguridad que tenga cada estado ${ }^{18}$. Baldwin la reformula en "baja probabilidad de daño"19 ya que se pueden incluir otros elementos que no se pueden controlar y que afecten a la seguridad (como desastres naturales) más allá del poder militar de otros estados. Wolfers intenta explicar la seguridad, $y$, por tanto, las políticas de seguridad de cada estado, identificando: a quién se protege si al estado o a los individuos, qué valores se quieren proteger, cuánta seguridad se quiere tener, cuáles son las amenazas, con qué medios, a qué coste y en cuánto tiempo ${ }^{20}$.

Aun así, empiezan a surgir otros conceptos como el de seguridad multidimensional (que abarca esferas no militares), el de seguridad cooperativa (la seguridad es fruto de la cooperación entre estados), o el de seguridad comprehensiva (la seguridad es mantener la independencia del estado y sus sociedades en cuanto a su identidad frente a fuerzas hostiles) ${ }^{21}$ que representaron una novedad, a pesar de que siguieran tomando como referente al estado22.

Después de la Guerra Fría, será Barry Buzan el que haga un análisis del concepto de seguridad en su libro People, States and Fear. Buzan identifica tres niveles de seguridad: los individuos, los estados y los sistemas internacionales; cinco sectores: el político, el militar, el económico, el social y el medioambiental; y lo que se podrían llamar aspectos o dimensiones de las políticas de seguridad.

Así en los tres niveles analizaríamos al estado que se identificaría como el referente principal; pero habría que preguntarse cuál es la naturaleza del estado ${ }^{23}$. Esto ayuda para entender

16 ABAD QUINTANAL, Gracia, “El concepto de seguridad: su transformación” en Comillas Journal of International Relations, $\mathrm{n}^{\circ}$ 4, 20 I 5, pp. 4I-5I.

17 Citado en: Ibídem, p. 42.

18 WOLFERS, Arnold, “National Security as an ambiguous symbol” en Political Science Quarterly, vol. 67, n 4, I 952 , pp. 48I-502.

19 BALDWIN, David A.,"The concept...”, op.cit., p. 20.

20 WOLFERS, Arnold, “National Security...”, op.cit., pp. 484-502.

2I BUZAN, Barry, “New Patterns of Global Security in the Twenty-First Century” en International Affairs, vol. 67, n 3, I 99I, pp. 43I-45I.

22 PÉREZ DE ARMIÑO, Karlos, "Seguridad humana y estudios críticos de seguridad: de la cooptación a la emancipación" en PÉREZ DE ARMIÑO, Karlos y MENDIA AZKUE, Irantzu (eds.), Seguridad Humana, aportes críticos al debate teórico y político, Tecnos, Madrid, 20I3, p. 28. Actualmente se habla de la seguridad transdominio y defensa comprehensiva incorporando más apellidos al concepto de seguridad, que sigue sin estar definido.Ver GARCÍA CANTALAPIEDRA, David,"Hacia un nuevo concepto de seguridad en un espacio multidominio: complejidad, guerra y seguridad transdominio” en Documento de Opinión IEEE, n 85, 20I9: http://www.ieee.es/Galerias/fichero/docs_opinion/20I9/ DIEEEO85_20I9DAVGAR_seguridad.pdf [consultado el II de diciembre de 2019].

23 STONE, Marianne, "Security according to Buzan: a comprehensive security analysis" en Security Discussion Papers Series I, Groupe d'Etudes et 
la seguridad de entidades más amplias y complejas que el individuo en sí, porque el individuo, el segundo nivel, puede entender su seguridad en términos de salud, vida, estatus, libertad, bienestar, por ejemplo, pero "no se puede copiar y pegar la seguridad individual en la seguridad del estado" 24 . De hecho, Buzan considera tres puntos de un triángulo para referirse al estado: la idea del estado, su composición física y las instituciones como partes interconectadas y el estudio de las relaciones entre ellas daría una visión más completa de la problemática de la seguridad nacional. El tercer nivel serían los organismos internacionales con un carácter mucho más amorfo y ambiguo que los estados.

Respecto a los cinco sectores que identifica Buzan en su New patterns of global security in the Twenty first century ${ }^{25}$ explica que no actúan independientemente, sino que cada uno enfoca un aspecto de la problemática de la seguridad y es una manera de ordenar prioridades, pero todos están interconectados. En función de estos sectores se tienen unas amenazas en cuenta por encima de otras. Hablaría de amenazas (y seguridades): militares, políticas, económicas, societales, y, medioambientales.

A la hora de tener en cuenta el análisis de políticas públicas de seguridad en el caso del estado, éste tiene que analizar las amenazas y las vulnerabilidades que le pueden situar en una posición de inseguridad (partiendo de que la situación de seguridad absoluta es inexistente). Es aquí donde Buzan introduce dos dimensiones: la interna y la externa, según en qué factores se centre la política de seguridad estatal. Si se centra en lo externo buscará terminar con las amenazas en su fuente de origen, lo que se llama una estrategia internacional de seguridad; si, por el contrario, se centra en lo interno tratará de disminuir las vulnerabilidades del estado lo que él llama una estrategia de seguridad nacional. Sin embargo, lo óptimo sería encontrar una política que mezclara elementos de la estrategia de seguridad nacional con elementos de la seguridad internacional ${ }^{26}$.

A modo de conclusión de este epígrafe, encontramos que la Guerra Fría ha servido como punto de inflexión para los estudios críticos de seguridad y de las Relaciones Internacionales en la evolución del concepto de seguridad. Antes de la Guerra Fría, la mayoría de autores hablaban de una seguridad estatocéntrica entendida en términos militares. Durante la Guerra Fría se empieza a intuir la interdependencia de las distintas áreas de la seguridad más allá de la estrictamente militar y se introducen nuevos conceptos como la seguridad multidimensional y la seguridad cooperativa. Pero es a partir de la caída del muro de Berlín cuando Buzan plantea cuestiones que después retomarían autores como Emma Rothschild en 1995 y Baldwin en 1997, que amplían el concepto en cuanto a las amenazas y profundizan en cuanto al sujeto de seguridad. Es, precisamente, esta ampliación y este planteamiento en el que se relaciona la economía con la seguridad y por tanto el desarrollo, lo que permite la aparición de nuevos conceptos que, además, tengan en cuenta el cambio en el paradigma internacional, donde los conflictos ya no son externos sino internos ${ }^{27}$.

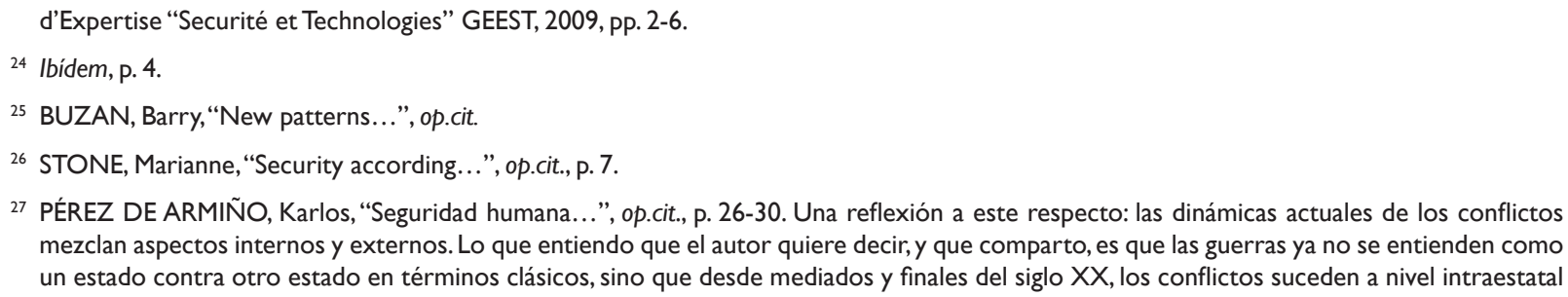
mezclan aspectos internos y externos. Lo que entiendo que el autor quiere decir, y que comparto, es que las guerras ya no se entienden como un estado contra otro estado en términos clásicos, sino que desde mediados y finales del siglo XX, los conflictos suceden a nivel intraestatal 
Estos nuevos conceptos siguen apareciendo fruto de la indefinición del término de seguridad, por un lado, se reflejan en los esfuerzos para seguir definiendo la seguridad como seguridad transdominio ${ }^{28} ; y$, por otro lado, están los conceptos que intentan proponer respuestas a qué hacer frente a estos nuevos conceptos de seguridad como el de resiliencia ${ }^{29} \circ$ el de defensa comprehensiva ${ }^{30}$.

\section{I.2 Cambio en el paradigma de la seguridad: seguridad humana, ¿un nuevo concepto?} No es hasta que con la caída del muro de Berlín en 199I se entiende por finalizada la Guerra Fría y cambia el paradigma, ¿qué es una amenaza a la seguridad? Ha desaparecido la posibilidad de la destrucción mutua asegurada fruto de la carrera armamentística y la amenaza de la guerra nuclear entre ambos bloques, $y$ es cuando empiezan a ganar mayor peso otros enfoques de la seguridad.

Paralelamente se habían ido sucediendo en el mundo una serie de conflictos y masacres, especialmente en la década de los ochenta y noventa, fruto de factores socioeconómicos más que militares y que afectaban más a las personas que al estado, que muchas veces es o promotor de la violencia o no puede proteger a su población. Estas guerras sirvieron para que tomaran peso unas corrientes más preocupadas por la protección de las personas, la defensa de los derechos humanos y el estudio del vínculo entre desarrollo y seguridad, lo que daría pie al nacimiento de conceptos como el de la responsabilidad de proteger o el de seguridad humana, como se detallará en el epígrafe 2.2 .

Efectivamente, con la caída del muro de Berlín y el fin de la Guerra Fría el concepto de seguridad se complejiza porque no sólo se tiene en cuenta la seguridad nuclear, armamentística ( $y$ entendida por tanto en términos militares) entre los dos bloques ${ }^{31}$. El concepto tradicional de seguridad con el estado como referente es insuficiente teniendo en cuenta la interdependencia de los estados y la aparición de nuevos aspectos en el concepto de seguridad. El principal problema aquí reside en decidir dónde parar, ya que la seguridad de los individuos puede verse afectada de maneras muy diversas, $y$ la seguridad pasa a convertirse en sinónimo de todo lo considerado políticamente bueno y deseable ${ }^{32}$.

Uno de los conceptos relacionados con el de seguridad que pretende aglutinar las nuevas dimensiones de la misma a la que se hacía referencia en la sociedad internacional es, como decíamos, el de seguridad humana. Este concepto empieza a abrirse paso con el Instituto

\footnotetext{
donde se mezclan fuerzas estatales con grupos armados del estado en cuestión, y otros estados participan según su interés geopolítico y económico en las mismas, bien a través de financiación a los actores en conflicto o enviando su propio contingente (casos concretos de conflictos de los últimos años en Afganistán, Irak, Siria, Libia, Yemen, Venezuela...).

28 Este nuevo concepto habla del control del dominio físico, eliminación por completo de lo interno y externo, y se difuminan las identidades estatales y del resto de actores.

${ }^{29}$ Es un enfoque más propio de la cooperación al desarrollo y de la acción humanitaria que habla de capacitar a las poblaciones para prevenir desastres y fomentar un desarrollo sostenible e inclusivo. Está muy relacionado con el concepto de seguridad humana porque se centra en la población, y también con el de seguridad porque los desastres como los conflictos son catalogados como amenazas a la seguridad.

30 Este concepto, como su nombre indica, hace referencia a la defensa desde todos los dominios, a saber: militar, civil, económico, social, psicológico, y digital. En este punto resaltar también la importancia del soft power que cobra más importancia en alguno de estos dominios sobre el hard power (o militar).

${ }^{31}$ Baldwin a este respecto señala que no se trata tanto de que no se tuviera en cuenta el concepto de seguridad durante la Guerra Fría, sino que los estudios de seguridad se centraban en las fuerzas militares y no en la seguridad en sí; lo que se opone a las explicaciones de Buzan de por qué no se ha estudiado sobre el concepto de seguridad.

32 WAEVER, Ole, “Securitization and Desecuritization” en LIPSCHUTZ, Roonie (dir.), On Security, Columbia University Press, Nueva York, 1995 , p. 47.
} 
Internacional de Estudios Estratégicos Humanos de Londres que en 1992 llama a la búsqueda de un nuevo concepto de seguridad que tenga en cuenta cualquier amenaza a la seguridad de naturaleza política, estratégica, económica, social o ecológica ${ }^{33}$. El testigo lo recoge el Programa de las Naciones Unidas para el Desarrollo (PNUD) con el capítulo dos del Informe sobre Desarrollo Humano de 1993: Nuevas dimensiones de la seguridad humana, que se considera la primera gran articulación del término ${ }^{34}$.

La respuesta a la pregunta, ¿un nuevo concepto? que da título a este epígrafe se tratará de responder desde la definición de seguridad humana que realiza el PNUD. En la redacción del Informe sobre seguridad humana destaca la figura de Mahbub UI Haq que la introdujo como categoría para el desarrollo humano ${ }^{35}$. UI Haq identifica que, para que haya desarrollo humano, se necesita estabilidad. El vínculo entre seguridad y desarrollo se hace patente también en los círculos políticos y académicos, y, por tanto, incluir un nuevo concepto de seguridad alejado de la fuerza militar y que tuviera como objeto el bienestar de las personas es necesario para cambiar la percepción y asegurar el desarrollo humano como proceso. El problema, o problemas, es que la seguridad humana no fue claramente definida, existen diferentes corrientes (la ampliada y la restringida) y, además, no es un nuevo concepto como tal.

EI PNUD define la seguridad humana como alternativa al tradicional concepto de seguridad, y con el contexto de post Guerra Fría, más concretamente, como alternativa al de seguridad nuclear de la siguiente manera:

"El mundo nunca podrá disfrutar de la paz, a menos que los seres humanos tengan seguridad en sus vidas cotidianas. Tal vez en el futuro los conflictos se produzcan con frecuencia dentro de un mismo país y no entre distintos países; y los orígenes de esos conflictos tal vez estén profundamente enraizados en las recientes disparidades y privaciones socioeconómicas. En esas circunstancias la búsqueda de seguridad humana debe efectuarse a través del desarrollo y no mediante las armas" 36 .

También afirma que:

"Durante un tiempo demasiado largo, la seguridad se ha equiparado a la protección frente a las amenazas a las fronteras de un país. Durante un tiempo demasiado largo, los países han tratado de armarse a fin de proteger su seguridad. Actualmente, para la mayoría de las personas, el sentimiento de inseguridad se debe más a las preocupaciones acerca de la vida cotidiana que al temor de un cataclismo en el mundo" ${ }^{37}$.

${ }^{33}$ ABAD QUINTANAL, Gracia, “El concepto de seguridad...", op.cit., p. 46.

${ }^{34}$ MARTIN, Mary y OWEN, Taylor, "The second generation of human security: lessons from the UN and EU experience" en International Affairs, vol. 86, $n^{\circ} 1,2010$, p. 213.

35 DUBOIS MIGOYA, Alfonso, "El concepto de seguridad humana desde el desarrollo humano: la predictibilidad del bienestar como exigencia" en PÉREZ DE ARMIÑO, Karlos y MENDIA AZKUE, Irantzu (eds.), Seguridad Humana, aportes críticos al debate teórico y politico, Tecnos, Madrid, 2013, p. $58-60$

${ }^{36}$ PNUD, Informe sobre Desarrollo Humano 1994, Naciones Unidas, 1994, p. I: http://hdr.undp.org/sites/default/files/hdr_1994_es_completo_ nostats.pdf [Consultado el 20 de septiembre de 2019]

37 Ibídem, p. 3. 
Efectivamente, trata de desmarcarse del anterior concepto de seguridad centrado en los conflictos entre estados. Lo que busca es pivotar de la seguridad del territorio a la seguridad de la población, y, de la seguridad mediante armas a la seguridad mediante el desarrollo humano. El PNUD define la seguridad humana como universal, interdependiente, preventiva y centrada en el ser humano. Los componentes o áreas de actuación serían la seguridad económica, alimentaria, de la salud, ambiental, personal, comunitaria y política. Estos componentes hacen también referencia a la tipología de las amenazas a esa seguridad humana.

La tipología de las amenazas y las características de la seguridad humana, así como el propio concepto son abstractos y construcciones político sociales. Esto hace difícil su operacionalización y posterior puesta en práctica. Es verdad que el propio Informe establece unas propuestas como la reducción anual del 3\% el gasto militar mundial; el pacto 20:20 para el desarrollo humano; o el Fondo Mundial de la Seguridad Humana a partir de impuestos internacionales (como el impuesto Tobin); pero, su falta de puesta en práctica, así como la esencia abstracta del concepto dificulta su consecución. De hecho, el propio Informe reconoce que es un concepto difícil de precisar y deja "sin cerrar su definición"38.

Por otro lado, existen dos corrientes que pugnan por un concepto amplio o restringido de seguridad humana. El primero integra la libertad respecto de la necesidad y el segundo que se limita a las amenazas contra la integridad física de las personas. Esto genera claramente una falta de consenso que inevitablemente va en detrimento del uso del concepto de seguridad humana.

Por último, y, respondiendo a la pregunta que se plantea en el epígrafe, por mucho que la seguridad humana trate de desentenderse del concepto tradicional de seguridad, lo cierto es que podría verse como una evolución del mismo. La palabra "humana" sigue yendo detrás de la palabra "seguridad" y las connotaciones que esto implica afectan directamente al establecimiento de unas u otras políticas, en este caso securitarias. Se podría entrar aquí en un debate en cuanto a la ampliación de las áreas que han pasado a formar parte de las políticas de seguridad y de otras que han dejado de serlo39. Pero, aun así, hay que reconocer que sigue siendo una versión mejorada del concepto de seguridad tradicional.Así pues, a pesar de los esfuerzos desde Naciones Unidas y de sus impulsores de relacionarla con los derechos humanos, sigue estando vinculada al concepto de seguridad entendido como una relación de poder ${ }^{40}$ entre estados. Esto significa que los gobiernos utilizan la palabra seguridad como un paraguas bajo el que situar lo que consideren una amenaza ${ }^{41}$ en ese momento, en aras de la seguridad nacional (e internacional), que sigue siendo, efectivamente, un cajón de sastre donde se incluyen aspectos como el terrorismo o el cambio climático.

Es por todo lo arriba expuesto que la seguridad humana no se consigue desvincular del todo del concepto de seguridad, y, por tanto, no es un nuevo concepto en sí mismo.Y la falta de precisión en la definición de qué es seguridad, unida a la falta de precisión también del propio concepto de seguridad humana han hecho que: primero, se entremezcle con otros conceptos parecidos, también vinculados a la defensa de los derechos humanos, como el de la responsabilidad

38 DUBOIS MIGOYA,Alfonso, “El concepto de seguridad...”, op.cit., p. 65.

39 WAEVER, Ole, “Securitization and...”, op.cit., p. 47-86; STONE, Marianne “Security according...”, op.cit., pp. 8-9.

40 DAHL, Robert A.,"The concept of power” en Behavioral Science, vol. 2, n 3, I957, pp. 20 I-2I 5.

${ }^{41}$ Ver el concepto “securitization” y “macro-securitization” de Ole Waever y Barry Buzan, respectivamente. 
de proteger y, segundo, otros conceptos desvinculados de la parte "seguridad" y que ponen el foco en la acción de las personas por y en su comunidad para promover el desarrollo, como el de resiliencia, ganen en detrimento del uso de la seguridad humana.

\section{Seguridad humana: un enfoque de derechos}

El concepto de seguridad humana engloba la protección o más bien, la securitización que se mencionaba anteriormente de aspectos que antes estaban exclusivamente en la temática de los derechos humanos. Es también después de los atentados del II-S donde este proceso de securitización se amplía, incluyéndose dentro del apartado "amenazas a la seguridad internacional" el terrorismo internacional, la degradación del medioambiente, la vulneración de los derechos humanos, la pobreza, y las enfermedades infecciosas ${ }^{42}$.

Desde el momento en el que la vulneración de los derechos humanos pasa a ser considerada amenaza a la seguridad internacional deja de tener tanto sentido el concepto de seguridad humana de años atrás. Esto, unido al auge de otros conceptos en la década de los noventa como el de la responsabilidad de proteger, que se explicará en el epígrafe 2.2, hace que el significado y la operacionalización de la seguridad humana sea todavía más difusa. De hecho, los conceptos se entremezclan desde los documentos de las Naciones Unidas que se publican esos años. El Informe, por ejemplo, Un concepto más amplio de la libertad: desarrollo, seguridad y derechos humanos para todos ${ }^{43}$ de 2005 habla de desarrollo humano, de protección de los derechos humanos en situaciones de conflicto y posconflicto como algo necesario para la estabilidad (seguridad humana), de la responsabilidad de la comunidad internacional de garantizar esa seguridad y de esa protección a las personas sean de donde sean (responsabilidad de proteger).

Sin embargo, la securitización de los derechos humanos puede tener consecuencias positivas y también negativas. El problema de los conceptos como seguridad o seguridad humana es que su falta de delimitación hace que puedan ser utilizados según los intereses, en este caso, de los gobiernos.Así pues, aspectos que antes no se consideraban amenazas a la seguridad internacional o nacional, ahora sí que se consideran y, mientras exista esa falta de delimitación, podrán seguir incluyéndose tantas otras como se considere necesario, como ya advertían Buzan y Ole Waever ${ }^{44}$ entre otros.

\section{I Vinculación con los derechos humanos: aspectos positivos y negativos}

Al vincular el concepto de seguridad con los derechos humanos, necesariamente se está hablando de seguridad humana. La seguridad humana recordemos que engloba la libertad respecto de la necesidad y la libertad respecto del miedo, es decir, que las personas se sientan libres, dignas y seguras en sus vidas cotidianas. Por lo tanto, al hablar de proteger el medioambiente para proteger a las personas, de una salud y alimentación necesaria, de libertad de expresarse sin miedo, etcétera se están protegiendo aspectos que antes eran exclusivos de la Declaración Universal de los Derechos Humanos. Y, atención, se protegen desde una perspectiva de seguridad. Esto significa

\footnotetext{
42 Naciones Unidas, Un mundo más seguro, la responsabilidad que compartimos, 2004: https://undocs.org/es/A/59/565 [Consultado del 20 de septiembre 2019].

43 NACIONES UNIDAS, Un concepto más amplio de la libertad: desarrollo, seguridad y derechos humanos para todos, 2005: https://documents-dds-ny. un.org/doc/UNDOC/GEN/N05/270/8I/PDF/N052708I.pdf [Consultado el 20 de septiembre 2019].

${ }^{44}$ Ver página 10 del presente artículo.
} 
que dentro de las políticas y acciones de seguridad pueden englobarse medidas que vayan para prevenir o paliar las malas condiciones socioeconómicas de las personas que no tengan seguridad humana.

Resulta interesante, siguiendo la línea de la falta de claridad de los conceptos, convertirlo en la siguiente reflexión partiendo de estas premisas: primero, cuando algo es considerado una amenaza para la seguridad internacional, se puede intervenir en otro país; segundo, la vulneración de los derechos humanos es considerada amenaza para la seguridad internacional; tercero, además, el concepto de la responsabilidad de proteger hace hincapié en, precisamente, la responsabilidad internacional de intervenir cuando el estado no puede o no quiere proteger a su población y darle seguridad.Teniendo estas tres premisas en cuenta: ¿se podría, teóricamente, intervenir en un país con una situación socioeconómica pobre desde la perspectiva de la seguridad? En teoría sí, pero existen ámbitos como la cooperación al desarrollo y la acción humanitaria que responden precisamente a esta pregunta. Pero, si entra dentro del ámbito de la seguridad, con políticas de seguridad, ¿cuáles serían los medios para responder a esa amenaza internacional a la seguridad que es la pobreza? ¿Los propios de las políticas de seguridad, es decir, militares? Queda demostrado que los medios militares no son la solución para resolver la pobreza, así que claramente no. Sin embargo, estos argumentos se han utilizado para intervenir en países como en Irak en 2003 al hablar de la opresión del pueblo ${ }^{45}$. Lo que se puede deducir de esta reflexión es que existe un solapamiento de áreas, de conceptos, de políticas que hacen complicado estudiar y delimitar la acción en aras de la seguridad internacional y/o nacional desde una sola perspectiva.

Unir, entonces, las áreas de seguridad, desarrollo humano, protección de derechos humanos en la seguridad humana tiene aspectos positivos y negativos. En cuanto a los positivos se puede hablar del carácter de urgencia que se le atribuye a cualquier elemento que pueda ser considerado amenaza a la seguridad. Efectivamente, ese carácter de urgencia que lleva implícita la palabra "amenaza" puede "aumentar la probabilidad de que la comunidad internacional reaccione con medidas que van más allá de la mera muestra de indignación frente a situaciones caracterizadas por una desprotección a gran escala o por procesos y sucesos que la causen" ${ }^{46}$.

Sin embargo, también existen efectos negativos que recoge, muy claramente Jessica Almqvist ${ }^{47}$. La autora señala dos concretamente: el empeoramiento de la protección universal de los derechos humanos y la marginalización de los órganos competentes en materia de protección de derechos humanos frente a otros encargados de la seguridad. Son dos efectos negativos lógicos derivados de la securitización desde el I I-S y en concreto desde la inclusión del terrorismo global como amenaza internacional ${ }^{48}$. En concreto, la llamada guerra contra el terrorismo ha hecho que numerosas materias pertenecientes a otras políticas se filtren también en el cajón de las políticas de seguridad. Un ejemplo de esto es la política migratoria y la política de seguridad en la Unión Europea, o la base de Guantánamo, cuya explicación sobre cómo están vinculadas y cuál es el

\footnotetext{
45 A este respecto, leer la Resolución 1483 del Consejo de Seguridad de las Naciones Unidas donde se legitima la intervención en Irak con el fin de destruir las armas de destrucción masiva y devolver "la soberanía al pueblo iraqui".

46 ALMQVIST, Jessica, “El futuro de la seguridad humana: una reflexión desde los derechos humanos” en PÉREZ DE ARMIÑO, Karlos y MENDIA AZKUE, Irantzu (eds.), Seguridad Humana, aportes críticos al debate teórico y político, Tecnos, Madrid, 20I3, p. I59.

47 Ibídem, pp. 161-162.

48 STONE, Marianne, “Security according...”, op.cit., pp. 8-9.
} 
proceso de detención respectivamente, daría para dos artículos cuanto menos.

La conclusión de estos aspectos positivos y negativos es que el concepto de seguridad se ha convertido en un concepto holístico y en constante evolución. Ya no se puede hablar de la seguridad tradicional, pero tampoco de seguridad humana exclusivamente. Lo que empezó como una alternativa al concepto estatocéntrico y militar de seguridad se ha convertido en una dimensión más del término seguridad, pues se han incluido los aspectos y dimensiones que comprendían la seguridad humana en el ideario securitario de los países a través, precisamente, de la securitización de las materias, entre ellas las de los derechos humanos. Esto, como se desarrollará en la conclusión, pone de manifiesto el peligro de entremezclar políticas de seguridad con los derechos humanos, porque puede suponer que se prime la seguridad, entendida en un sentido más clásico y tradicional, sobre los derechos humanos.

\subsection{Vinculación con la responsabilidad de proteger}

Como se ha mencionado al comienzo de este epígrafe, el concepto de seguridad humana está íntimamente relacionado con el de responsabilidad de proteger. Es interesante dedicarle un apartado a su estudio porque la responsabilidad de proteger es otro concepto en desuso, como el de seguridad humana, debido en parte a las mismas causas (falta de delimitación del concepto, ausencia de consenso internacional, su utilización en casos obedeciendo a intereses geopolíticos).

Las cuestiones previas al nacimiento y desarrollo de la responsabilidad de proteger están interrelacionadas con las de la seguridad humana. El aumento del número de bajas civiles en los conflictos armados, unido a la extensión de los derechos humanos allanó el camino para que en la comunidad internacional se propusieran los conceptos de seguridad humana y soberanía como responsabilidad. Es precisamente, desde que la seguridad se entiende como integral y no sólo como la satisfacción de necesidades básicas ${ }^{49}$, que la defensa de los derechos humanos deja de estar, como se decía antes, exclusivamente en manos de un gobierno en concreto, sino que es responsabilidad de toda la comunidad internacional.

Paralelamente la soberanía se entiende como responsabilidad. Esto surge por la definición del estado como territorio, gobierno, población y soberanía. La población es, por tanto, parte esencial, $y$, el estado expresado a través de ese gobierno tiene que proteger a la población que lo representa. Esta idea es la base que Francis Deng ${ }^{50}$ utilizará para desarrollar la responsabilidad de proteger. La aparición de la seguridad humana es esencial también para desarrollar la doctrina de la responsabilidad de proteger porque se trata de justificar las intervenciones en otros países con una respuesta desde un punto de vista securitario y militar por motivos de vulneración de los derechos humanos.

En conclusión, la conjunción de cambios operados en el escenario internacional hace que la seguridad humana y la responsabilidad de proteger vayan unidas de la mano. En este caso, la seguridad humana, unida a la soberanía como responsabilidad y a los derechos humanos, junto con la voluntad de dar respuestas a una coherencia en el sistema legal internacional es lo que se

\footnotetext{
49 VALLEJO, Beatriz E., “La responsabilidad de proteger. Una nueva dimensión de la soberanía” en Oasis, n I 5, 2010 , pp. 7-32.

50 DENG, Francis et al., Sovereignity as responsibility: conflict management in Africa, Brooking Institution Press, Washington DC, 1996.
} 
plasmó en la doctrina de la responsabilidad de proteger ${ }^{51}$.

\section{Conclusiones}

En este epígrafe se tratará de dar respuesta a por qué se ha dejado de usar el concepto de seguridad humana, como se avanzaba en la introducción; $y$, qué significa, o puede significar, este cambio. El análisis de la evolución del término de seguridad, así como el de seguridad humana y su vinculación con los derechos humanos, parte esencial del concepto, ha servido para identificar tres causas concretas (y es posible que haya otras) a este respecto.A saber, la falta de delimitación del concepto lo convierte en un término de por sí inútil, en el sentido que no puede usarse con consenso, y, queda a merced de la politización, segunda causa identificada. Esta politización, derivada precisamente de la falta de claridad en la definición del concepto, está estrechamente relacionada con la securitización de otras materias, y con la propia perversión del término, donde se puede llegar incluso a violar derechos humanos tratando de justificar la seguridad humana de otras personas. Estas dos causas llevan aparejadas que, desde los campos de la acción humanitaria y de cooperación al desarrollo, se promueva el uso de otros términos, como resiliencia, que pongan el peso más en la población y menos en las acciones del gobierno, convirtiendo el concepto de seguridad humana en un enfoque desde el que responder más que un concepto en sí mismo. $A$ continuación, se explican con detalle las causas del fracaso en el uso del término de seguridad humana:

Primero, la falta de concreción del término. El término seguridad es una construcción político social amplia, multidimensional y viva. Por tanto, su definición se ajusta a la subjetividad de cada uno. Los intentos de definirla y delimitarla para poder analizarla y estudiarla, así como para comparar con otras políticas de seguridad han sido arduos, exhaustos, pero sin consenso, $y$, por tanto, no existe una definición clara y concreta de qué es seguridad.

De la misma manera que no se puede llegar a definir qué es seguridad, tampoco se puede, por los mismos motivos, encontrar una clara y delimitada definición de seguridad humana. De hecho, según qué autores puede tratarse de una dimensión de la seguridad, o un término totalmente alternativo, como proponía el PNUD.

Como se puede ver a lo largo del artículo, las diferentes clasificaciones de seguridad, las tipologías de amenazas, así como a los objetos que amenazan, varían según épocas, corrientes politológicas y filosóficas y autores. Esto implica que la diferente denominación de lo mismo depende del enfoque o del énfasis que se quiera dar predominando una dimensión sobre otra, o justificando las amenazas desde una teoría u otra: defensa de los derechos humanos o lucha contra el terrorismo internacional, por ejemplo.

Lo cierto es que la securitización de diferentes materias en el cajón de la seguridad ha restado valor a otros conceptos que se prestaran como alternativos, pues al final, todo puede considerarse amenaza a la seguridad, incluidas las amenazas que identificaba la seguridad humana como propias, lo que nos lleva al siguiente punto.

\footnotetext{
51 HERNÁNDEZ-GARCÍA, Nuria,“La Unión Europea ante la responsabilidad de proteger: una aproximación a través del análisis de sus misiones
} en el marco de la Política Común de Seguridad y Defensa” en Revista Deusto de Derechos Humanos, n 3, 20I8, pp. I03-I05. 
Segundo, su uso selectivo o politización. El hecho de la ampliación del término de seguridad en este siglo XXI donde se pone el foco de atención en la persona y no en el estado, es un arma de doble filo como se ha ido observando a lo largo del texto. Los gobiernos pueden incluir bajo el título de amenaza cualquier aspecto que se les ocurra que afecte a sus intereses nacionales y geoestratégicos. Esto lo que genera es una confusión con respecto al uso del término de seguridad humana, algo que también le pasó a la responsabilidad de proteger. Esta confusión con respecto a cuándo usarlo y cuándo no se traduce en una politización del término, debido al mal uso del mismo por los gobiernos, lo que inevitablemente le resta credibilidad. Y, cuando un término deja de tener credibilidad, precisamente por su uso selectivo e interesado, consecuencia de una falta de delimitación conceptual entre otros aspectos, deja de tener valor.

Por otro lado, precisamente la facilidad para securitizar materias, unido al uso selectivo de qué significa defender la seguridad humana de las personas, da lugar a incoherencias como violar los derechos humanos. Existen gobiernos en Europa que afirman proteger a su población de las amenazas de la inmigración, por ejemplo. Con acciones que sostienen defender la identidad y cultura de la población, los trabajos y la estabilidad socioeconómica de la población o garantizarles una integridad física sin terrorismo, justifican una política migratoria securitizada donde se violan derechos humanos en materia de asilo y refugio. He ahí la incoherencia, porque precisamente la seguridad humana es para todos, y se trata de defender y proteger los derechos humanos de todos. Sin embargo, como ocurría con el concepto tradicional de seguridad, utilizar esta palabra convierte cualquier política en urgente y necesaria. Lo que nos lleva a la siguiente causa identificada para justificar el deje del uso del término seguridad humana.

Tercero, el auge de otros conceptos dentro de lo que ahora es el enfoque de la seguridad humana. Como se mencionaba a lo largo del texto, la falta en la definición de qué es seguridad, unida a la falta de precisión también del propio concepto de seguridad humana ha hecho que: primero, se entremezcle con otros conceptos parecidos, también vinculados a la defensa de los derechos humanos, como el de la responsabilidad de proteger y segundo, se utilicen más otros conceptos desvinculados de la parte "seguridad" y que ponen el foco en la acción de las personas por y en su comunidad para promover el desarrollo, como el de resiliencia.

Según se señala en la Resolución 66/290 de la Asamblea General, “la seguridad humana es un enfoque que ayuda a los estados miembro a determinar y superar las dificultades generalizadas e intersectoriales que afectan a la supervivencia, los medios de subsistencia y la dignidad de sus ciudadanos" 52 . En la Resolución se exigen "respuestas centradas en las personas, exhaustivas, adaptadas a cada contexto y orientadas a la prevención que refuercen la protección y el empoderamiento de todas las personas" ${ }^{3}$. También hacen hincapié en la idea de que es un concepto diferente al de la responsabilidad de proteger, al de seguridad y que no entraña el uso coercitivo de la fuerza ni medios militares.

Es en esta Resolución donde encontramos el desmarque de la seguridad humana como

\footnotetext{
${ }^{52}$ A/RES/66/290 del 25 de octubre de 2012, Seguimiento del párrafo I 43, relativo a la seguridad humana, del Documento Final de la Cumbre Mundial 2005, Naciones Unidas: https://documents-dds-ny.un.org/doc/UNDOC/GEN/NI I/476/25/PDF/NI I 47625.pdf?OpenElement [Consultado el 21 de septiembre de 2019].

${ }^{53}$ Ibíd., punto 3.b).
} 
concepto y hablamos de la seguridad humana como enfoque. Este enfoque supondría un marco de análisis y planificación que permite responder de manera amplia y preventiva a los problemas "generados por conflictos, exclusión social y la pobreza extrema o que los provocan" 54 . En resumen, busca tener en cuenta, a la hora de dar respuestas a problemas que afecten a la vida de las personas, la relación entre paz y seguridad y desarrollo y derechos humanos. De esta manera el objetivo último de este enfoque es que las personas vivan libres de temor, sin miseria y con dignidad.

La seguridad humana se aplica, entonces, de manera de enfoque, como un prisma para responder ampliamente, como se mencionaba antes. Precisamente por el carácter preventivo que se menciona en la Resolución $66 / 290$, el objetivo es proteger y empoderar a las personas y a las comunidades para que puedan responder con resiliencia ante una amplia gama de amenazas.

La seguridad humana se convierte en otro cajón de sastre, desde el que analizar y planificar respuestas a diferentes contextos que afecten a la vida de las personas ${ }^{55}$. Precisamente esto último, el convertirse en un cajón de sastre, es la principal consecuencia del cambio de hacer referencia a la seguridad humana como concepto a utilizarla como enfoque. Esto respondería a qué significa el deje en el uso de seguridad humana como tal,y es que se convierte en un modo de hacer más que en un concepto con entidad en sí mismo.

Como conclusión final, el término seguridad humana ha dejado de usarse como justificación o alternativa a la seguridad. Se trata de un enfoque holístico y de carácter preventivo y no militar que busca mejorar la vida de las personas. Para ello, el objetivo es empoderar a las personas y comunidades a través de lo que se conoce como resiliencia. Sin embargo, desde Naciones Unidas y otros organismos, se sigue promoviendo su uso a modo de comodín para justificar las acciones que se enmarcarían en programas de cooperación al desarrollo, acción humanitaria o en intervenciones en conflictos. Se trata, pues, de una perspectiva que hay que aplicar en cualquier situación atendiendo al contexto particular y teniendo en cuenta la relación entre paz, seguridad, derechos humanos y desarrollo sostenible e inclusivo. Este modus operandi se relaciona inevitablemente con el resto de estrategias de las Naciones Unidas, donde nacía el concepto, con el mismo fin de mejorar la vida de las personas como la Agenda 2030 y los Objetivos de Desarrollo Sostenible (ODS). Así pues, el enfoque de la seguridad humana supone: primero que el concepto de seguridad humana en sí sea papel mojado; $y$, segundo, no le dota de efectividad, porque no hay una guía de control sobre si se aplica o no el enfoque de manera correcta, puesto que no está definido (volvemos a lo mismo).

A modo de conclusión de este trabajo advertiría del peligro de entremezclar las políticas o los conceptos que tienen que ver con la seguridad y con los derechos humanos. El resultado de entremezclarlos sería desdibujar la línea que separa seguridad y derechos y libertades de manera que pudieran violarse estas últimas en favor de lo primero. Por lo tanto, propondría: primero, no negar la obviedad de la complejidad de la definición de un término como es la seguridad; segundo,

\footnotetext{
${ }^{54}$ Fondo Fiduciario para las Naciones Unidas, La consecución de la Agenda 2030, Naciones Unidas: https://www.un.org/humansecurity/es/ agenda-2030/ [Consultado el 21 de septiembre de 2019].

55 Fondo Fiduciario para las Naciones Unidas, Qué es la seguridad humana, Naciones Unidas: https://www.un.org/humansecurity/es/what-ishuman-security/ [Consultado el 21 de septiembre de 2019].
} 
resaltar la seguridad como algo multidimensional, por lo que atañe a todas las esferas de la vida de los individuos, y multiinfluenciada, porque es afectada y modificada por diferentes actores de una manera directa, indirecta, transparente o encubierta ${ }^{56}$; tercero, utilizar los documentos de las Naciones Unidas, la Agenda 2030 y los ODS como hojas de ruta primando siempre la observancia de los derechos humanos y el desarrollo sostenible frente a cualquier otro aspecto.

\section{Bibliografía}

A/RES/66/290 del 25 de octubre de 2012, Seguimiento del párrafo 143, relativo a la seguridad Humana, del Documento Final de la Cumbre Mundial 2005, Naciones Unidas: https://documents-dds-ny.un.org/doc/UNDOC/GEN/ NI I/476/25/PDF/NI I47625.pdf?OpenElement [Consultado el 2 I de septiembre de 2019].

ABAD QUINTANAL, Gracia, "El concepto de seguridad: su transformación" en Comillas Journal of International Relations, $\mathrm{n}^{\circ} 4$, 20I5, pp. 4I-5I.

AGUIAR MOLINA, Pablo y ALCALDEVILLACAMPA, Javier, "Seguridad Humana. Un marco de análisis" en Conflictos, politica y derecho, 20II, pp. 21 -52.

BALDWIN, David A.," "The concept of security” en Review of International Studies, n² 23, 1997, pp. 5-26.

BUZAN, Barry, "New patterns of global security in the Twenty- first century" en International Affairs, vol. 67, n 3, 1991.

DAHL, Robert A.,"The concept of power" en Behavioral Science, vol. 2, n 3, 1957, pp. 20I-2I5.

DENG, Francis et al. Sovereignity as responsibility: conflict management in Africa, Brooking Institution Press, Washington DC, 1996.

Fondo Fiduciario para las Naciones Unidas, La consecución de la Agenda 2030, Naciones Unidas: https://www.un.org/ humansecurity/es/agenda-2030/ [Consultado el 2I de septiembre de 2019].

Fondo Fiduciario para las Naciones Unidas, Qué es la seguridad humana, Naciones Unidas: https://www.un.org/ humansecurity/es/what-is-human-security/ [Consultado el 2I de septiembre de 2019].

GARCÍA CANTALAPIEDRA, David,"Hacia un nuevo concepto de seguridad en un espacio multidominio: complejidad, guerra y seguridad transdominio" en Documento de Opinión IEEE, n 85, 2019: http://www.ieee.es/Galerias/ fichero/docs_opinion/2019/DIEEEO85_2019DAVGAR_seguridad.pdf [consultado II/diciembre/2019].

HERNÁNDEZ-GARCIIA, Nuria, "La Unión Europea ante la responsabilidad de proteger: una aproximación a través del análisis de sus misiones en el marco de la Política Común de Seguridad y Defensa" en Revista Deusto de Derechos Humanos, ${ }^{\circ}$ 3, 2018, pp. $103-105$.

MARTIN, Mary y OWEN,Taylor,"The second generation of human security: lessons from the UN and EU experience" en International Affairs, vol. 86, $\mathrm{n}^{\circ}$ I, 2010 , p. 2 I3.

Naciones Unidas, Un concepto más amplio de la libertad: desarrollo, seguridad y derechos humanos para todos, 2005: https:// documents-dds-ny.un.org/doc/UNDOC/GEN/N05/270/8I/PDF/N052708I.pdf?OpenElement [Consultado el 20 de septiembre 2019].

Naciones Unidas, Un mundo más seguro, la responsabilidad que compartimos, 2004: https://undocs.org/es/A/59/565 [Consultado del 20 de septiembre 2019].

OPPENHEIM, Felix E., "The language of political inquiry: problems of clarification" en GREENSTEIN, Fred y POLSBY, Nelson (eds.), Political Science: Scope and Theory, Addison-Wesley, Reading, 1975, p. 284.

PÉREZ DE ARMIÑO, Karlos y MENDIA AZKUE, Irantzu (eds.), Seguridad Humana, aportes críticos al debate teórico y político, Tecnos, Madrid, 2013.

PNUD, Informe sobre Desarrollo Humano 1994, Naciones Unidas, 1994, p. I: http://hdr.undp.org/sites/default/files/ hdr_1994_es_completo_nostats.pdf [Consultado el 20 de septiembre de 2019].

ROTHSCHILD, Emma, "What is security?" en Daedalus, vol. I24, n 3, 1995, pp. 53-98.

STONE, Marianne, "Security according to Buzan: a comprehensive security analysis" en Security Discussion Papers Series I, Groupe d'Etudes et d'Expertise "Securité et Technologies" GEEST, 2009, p. 2-6.

VALLEJO, Beatriz E.,"'La responsabilidad de proteger. Una nueva dimensión de la soberanía" en Oasis, n I5, 2010, pp. 7-32.

WAEVER, Ole, "Securitization and Desecuritization" en LIPSCHUTZ, Roonie (dir.), On Security, Columbia University Press, Nueva York, 1995, p. 47.

WOLFERS, Arnold, “National Security as an ambiguous symbol” en Political Science Quarterly, vol. 67, n 4, 1952, pp. $48 \mathrm{I}-502$. el contrario, hacerlo de manera transparente sería hacer referencia al conflicto de intereses entre Rusia y China en el Consejo de Seguridad de Naciones Unidas o la polarización latinoamericana en el Grupo de Lima con el caso de Venezuela. 


\section{RELACIONES INTERNACIONALES}

Revista académica cuatrimestral de publicación electrónica Grupo de Estudios de Relaciones Internacionales (GERI)

Universidad Autónoma de Madrid, España

https://revistas.uam.es/relacionesinternacionales

ISSN 1699 - 3950

f facebook.com/RelacionesInternacionales

3. twitter.com/RRInternacional

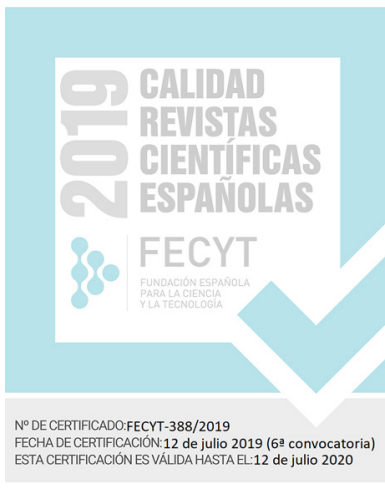

